\title{
Curvature estimation from a volume-of-fluid indicator function for the simulation of surface tension and wetting with a free- surface lattice Boltzmann method
}

Citation for published version (APA):

Bogner, S., Rüde, U., \& Harting, J. (2016). Curvature estimation from a volume-of-fluid indicator function for the simulation of surface tension and wetting with a free-surface lattice Boltzmann method. Physical Review E, 93(4), [043302]. https://doi.org/10.1103/PhysRevE.93.043302

DOI:

10.1103/PhysRevE.93.043302

Document status and date:

Published: 01/04/2016

Document Version:

Publisher's PDF, also known as Version of Record (includes final page, issue and volume numbers)

Please check the document version of this publication:

- A submitted manuscript is the version of the article upon submission and before peer-review. There can be important differences between the submitted version and the official published version of record. People interested in the research are advised to contact the author for the final version of the publication, or visit the $\mathrm{DOI}$ to the publisher's website.

- The final author version and the galley proof are versions of the publication after peer review.

- The final published version features the final layout of the paper including the volume, issue and page numbers.

Link to publication

\footnotetext{
General rights

- You may freely distribute the URL identifying the publication in the public portal. follow below link for the End User Agreement:

www.tue.nl/taverne

Take down policy

If you believe that this document breaches copyright please contact us at:

openaccess@tue.nl

providing details and we will investigate your claim.
}

Copyright and moral rights for the publications made accessible in the public portal are retained by the authors and/or other copyright owners and it is a condition of accessing publications that users recognise and abide by the legal requirements associated with these rights.

- Users may download and print one copy of any publication from the public portal for the purpose of private study or research.

- You may not further distribute the material or use it for any profit-making activity or commercial gain

If the publication is distributed under the terms of Article 25fa of the Dutch Copyright Act, indicated by the "Taverne" license above, please 


\title{
Curvature estimation from a volume-of-fluid indicator function for the simulation of surface tension and wetting with a free-surface lattice Boltzmann method
}

\author{
Simon Bogner" and Ulrich Rüde ${ }^{\dagger}$ \\ Lehrstuhl für Systemsimulation, Universität Erlangen-Nürnberg, Cauerstraße 11, 91054 Erlangen, Germany \\ Jens Harting ${ }^{\ddagger}$ \\ Forschungszentrum Jülich, Helmholtz-Institut Erlangen-Nürnberg (IEK-11), Fürther Straße 248, 90429 Nürnberg, Germany \\ and Department of Applied Physics, Technische Universiteit Eindhoven, P. O. Box 513, 5600 MB Eindhoven, The Netherlands \\ (Received 25 September 2015; revised manuscript received 10 February 2016; published 1 April 2016)
}

\begin{abstract}
The free surface lattice Boltzmann method (FSLBM) is a combination of the hydrodynamic lattice Boltzmann method with a volume-of-fluid (VOF) interface capturing technique for the simulation of incompressible free surface flows. Capillary effects are modeled by extracting the curvature of the interface from the VOF indicator function and imposing a pressure jump at the free boundary. However, obtaining accurate curvature estimates from a VOF description can introduce significant errors. This article reports numerical results for three different surface tension models in standard test cases and compares the according errors in the velocity field (spurious currents). Furthermore, the FSLBM is shown to be suited to simulate wetting effects at solid boundaries. To this end, a new method is developed to represent wetting boundary conditions in a least-squares curvature reconstruction technique. The main limitations of the current FSLBM are analyzed and are found to be caused by its simplified advection scheme. Possible improvements are suggested.
\end{abstract}

DOI: 10.1103/PhysRevE.93.043302

\section{INTRODUCTION}

The free surface lattice Boltzmann method (FSLBM) [1] is a numerical method for the simulation of free surface flows combining a volume-of-fluid (VOF) approach [2-4] for interface tracking with the lattice Boltzmann method (LBM) [5-9] for hydrodynamics. We use the same definition of free surface flow as described in Refs. $[3,10]$, where it denotes a single-phase flow problem containing free boundaries instead of a two-phase flow problem. VOF methods follow the notion of a sharp interface representation, i.e., assuming hydrodynamic equations for the bulk of the flow and modeling the interface by boundary conditions or by a jump of flow parameters in one-fluid approaches for two-phase flows. This is in contrast to currently popular lattice Boltzmann multiphase approaches [6,11,12] (e.g., the color gradient model [13], the Shan-Chen model [14], and the free-energy model [15]) that are based on a diffusive interface assumption. In the FSLBM, the LBM is used only to approximate the incompressible Navier-Stokes equations for the liquid phase. With sharp interface simulation techniques, capillary effects need to be modeled explicitly in addition to the interface tracking. Altogether, there are three components on which the total accuracy of the method depends: the hydrodynamic solver (LBM), the interface tracking (advection of indicator function), and the surface tension model. While the accuracy of the LBM is well understood [16-18], only very few results have been reported addressing the accuracy of the FSLBM's advection scheme or its validity to simulate surface tension. Nevertheless, the approach has found numerous applications in surface tension-driven complex flow scenarios [19-23] and even

\footnotetext{
*simon.bogner@fau.de

${ }^{\dagger}$ ulrich.ruede@fau.de; http://www10.informatik.uni-erlangen.de

${ }_{\ddagger}^{\dagger}$ j.harting@fz-juelich.de; http://mtp.phys.tue.nl/
}

high-Reynolds-number flows [24,25]. Hence, in the present paper we evaluate the accuracy of the FSLBM in surface tension driven flows and also discuss existing limitations due to the original advection scheme. While conventional VOF implementations rely on geometric reconstruction to approximate the advection equation of the indicator function $[3,4]$, the FSLBM instead exploits the specific nature of the LBM. However, the present results in this work indicate that though this simplification reduces the algorithmic complexity significantly, it also comes with a comparably low accuracy.

The simulation of surface tension involves the extraction of curvature information from the interface defined through the fill level function [3,26-28]. This VOF indicator function is nonsmooth by definition, making the estimation of the interface curvature a nontrivial task. Numerical errors in the determination of interface stresses lead to the effect of undesired spurious currents that can invalidate or destabilize the computed solutions. We remark that if the surface stress is included as a force term in the momentum equation as in Ref. [26], then the incompatible discretization of pressure and indicator function gradients becomes an additional source for spurious currents [29]. The present model does not have this problem, because surface tension is imposed as a pressure jump at the boundary instead. Anyway, errors in the curvature estimation are reported as problematic - primarily because the grid convergence is poor unless more sophisticated approaches are employed. Modern VOF codes therefore rely on higherorder curvature estimation techniques to suppress this error $[28,30]$. However, these techniques require the use of larger stencils. This may be undesirable or is even impossible if only next-neighbor information is available, as, e.g., in some parallel computing environments. Hence, in this paper we review and present three different techniques to approximate the interface capillary tension from the fill level function data of a free surface code that are using local $3 \times 3 \times 3$ neighborhoods only. 
The first method is an adaption of the classic finitedifference (FD) model by Brackbill et al. [26] that uses finitedifference computations to obtain the interface curvature. As reported previously for this method, the magnitude of the spurious currents makes it impossible to simulate small capillary number flows correctly. Notice that in Ref. [26], the surface tension appears as a force term in the momentum equation and, further, that this force is smoothed out over several grid cells. Hence this model is also called continuous surface force model in the literature. In the FSLBM presented here, surface tension is included in the boundary condition instead.

The second model locally reconstructs the interface described by the VOF function as a continuous surface made up of triangles. From this triangular reconstruction (TR) the curvature information can be extracted. This method has first been presented in Ref. [31], and turns out to effectively reduce the error due to spurious currents, because the predicted curvature values are much more accurate.

The third method also involves a local reconstruction of the interface geometry. Based on a least squares approach a parabolic approximation to the interface is constructed in a local neighborhood of cells. From this least-squares reconstruction (LSQR) one obtains curvature estimates with a high accuracy. A similar approach has also been described in Ref. [32] as a fallback solution for a higher-order heightfunction technique. For the current paper, we have extended the approach to problems including adhesive boundary conditions. It is the only model in this study to achieve a second-order rate of convergence in the classic spherical bubble benchmark.

We also evaluate the boundary conditions needed to simulate the wetting behavior of solid surfaces for the FD and LSQR model. The original work by Brackbill et al. [26] discusses adhesive boundary conditions, which we adopted to the FSLBM context for this paper. Since curvature estimation based on finite differences often introduces larger errors, smoothing and filtering techniques are typically used and have been extended to adhesive boundaries in Ref. [33] for the simulation of low-capillary-number flows. Motivated also by the limited accuracy of the finite-difference approximation of curvature by finite differences, the authors of Refs. [10] and [34] switched to the height function technique [35,36] for the simulation of surface wetting. Some other approaches use level sets instead of the VOF method to represent the interface [37] or directly use non-Eulerian techniques (e.g., Ref. [38]). In this paper, we present the results obtained from the FSLBM in several simulations of wetting surfaces while studying alternative surface tension models. This allows a comparison between the FD approach to surface tension and the newly developed LSQR method.

\section{NUMERICAL METHOD}

Throughout this paper we assume a three-dimensional D3Q19 lattice Boltzmann model $[8,39]$ with $Q=19$ lattice velocity vectors $\mathbf{c}_{q}$ with $q=0, \ldots, Q-1$. We denote the LBM data [discrete particle distribution function (PDF)] by $\mathbf{f}=\left(f_{0}, f_{1}, \ldots, f_{Q-1}\right)$. The LBM data are defined for every node within the liquid subdomain $\Omega(t)$, and they follow the evolution equation

$$
\begin{aligned}
f_{q}\left(\mathbf{x}+\mathbf{c}_{q}, t+1\right) & =f_{q}^{\prime}(\mathbf{x}, t), \\
\mathbf{f}^{\prime}(\mathbf{x}, t) & =\mathbf{f}(\mathbf{x}, t)+\mathbf{C}(\mathbf{f}(\mathbf{x}, t)),
\end{aligned}
$$

where $\mathbf{C}$ is a collision operator and $\mathbf{f}^{\prime}$ is the postcollision distribution function. For the present paper, we have used the hydrodynamic two-relaxation-time (TRT) collision operator [40]. The TRT operator has two eigenvalues $\left(\lambda_{e}\right.$ and $\left.\lambda_{o}\right)$ controlling the relaxation of the even and odd parts of the PDF, respectively. Similarly to the popular LBGK model [39], the relaxation rate $\tau=-1 / \lambda_{e}$ controls the kinematic viscosity $v=c_{s}^{2}(\tau-1 / 2)$, with $c_{s}=1 / \sqrt{3}$ for the present D3Q19 model. The second parameter $\lambda_{o}$ is chosen to minimize the error at straight axis-aligned walls. The macroscopic variables of pressure $P(\mathbf{x}, t)=c_{s}^{2} \rho(\mathbf{x}, t)$ and velocity $\mathbf{u}(\mathbf{x}, t)$ are defined via moments of the distribution function,

$$
\begin{gathered}
\rho=\sum_{q=0}^{Q-1} f_{q}, \\
\rho \mathbf{u}=\sum_{q=0}^{Q-1} \mathbf{c}_{q} f_{q},
\end{gathered}
$$

at the respective node $\mathbf{x}$.

The FSLBM was first described in Ref. [1]. To track the interface position in simulations, a VOF approach introduces a fill level function $\varphi$ following an advection equation over time. The function $\varphi$ is defined for each finite volume (or cell) as the volume fraction filled with liquid. Only the flow within the liquid subdomain $\Omega(t)$, consisting of all cells $\mathbf{x}$ with positive fill level $\varphi(\mathbf{x})>0$, is simulated by means of the LBM. The remaining cells, referred to as gas cells, become temporarily inactive. Within the liquid subdomain $\Omega(t)$, we distinguish between liquid and interface cells. To count as an interface, the cell must have both liquid and gas cells in the direct neighborhood defined by the lattice model. Only the interface cells are allowed to have a fill level between 0 and 1. The interface cells are also used to compute the advection of the free surface in terms of the fill level function and to impose a free surface boundary condition on the LBM. In comparison to other VOF methods, where the advection of the fill level function is a nontrivial problem that needs to be solved in addition to the hydrodynamics, the FSLBM exploits the nature of the lattice Boltzmann equation, Eq. (1a) and Eq. (1b), to update the fill levels directly [1] and set

$$
\begin{aligned}
& \varphi(\mathbf{x}, t+1) \\
& =\varphi(\mathbf{x}, t)+\frac{\sum_{q=1}^{Q-1} k(\mathbf{x}, q) \cdot\left[f_{\bar{q}}^{\prime}\left(\mathbf{x}+\mathbf{c}_{q}, t\right)-f_{q}^{\prime}(\mathbf{x}, t)\right]}{\rho(\mathbf{x}, t+1)},
\end{aligned}
$$

where the coefficient $k(\mathbf{x}, q)$ is

$$
k(\mathbf{x}, q):= \begin{cases}0 & \text { if } \mathbf{x}+\mathbf{c}_{q} \text { is gas, } \\ \frac{1}{2}\left[\varphi\left(\mathbf{x}+\mathbf{c}_{q}\right)+\varphi(\mathbf{x})\right] & \text { if } \mathbf{x}+\mathbf{c}_{q} \text { is interface } \\ 1 & \text { if } \mathbf{x}+\mathbf{c}_{q} \text { is liquid, }\end{cases}
$$

which means that there is no mass flux to gas cells. 
The free surface boundary condition for the LBM at the interface cells is

$$
f_{\bar{q}}(\mathbf{x}, t+1)=f_{q}^{\mathrm{eq}}\left(\rho_{b}, \mathbf{u}_{b}\right)+f_{\bar{q}}^{\mathrm{eq}}\left(\rho_{b}, \mathbf{u}_{b}\right)-f_{q}^{\prime}(\mathbf{x}, t),
$$

for all directions $q$ pointing towards gas cells $\mathbf{x}+\mathbf{c}_{q} \notin \Omega(t)$. Hereby, $f_{q}^{\prime}$ denotes the postcollision distribution oriented towards the gas phase and $f_{q}^{\mathrm{eq}}\left(\rho_{b}, \mathbf{u}_{b}\right)$ is the equilibrium distribution function [1]. It can be shown that Eq. (4) approximates a free boundary condition with first-order spatial accuracy [41]. The two boundary condition parameters $\rho_{b}$ and $\mathbf{u}_{b}$ are the macroscopic pressure and velocity at the interface, respectively. The boundary value $\rho_{b}$ is defined as

$$
\rho_{b}=\frac{1}{c_{s}^{2}}\left(p_{g}+p_{s}\right),
$$

where $p_{g}(t)$ is the static pressure at the free surface and $p_{s}(\mathbf{x}, t)$ is the Laplace pressure. The latter depends on the local curvature of the interface by

$$
p_{s}(\mathbf{x})=2 \sigma \kappa(\mathbf{x})
$$

with a constant surface tension $\sigma$. We remark that in the original work of [1], Eq. (4) is applied for all $q$, oriented outwards with respect to the interface, i.e., $\mathbf{c}_{q} \cdot \mathbf{n} \leqslant 0$, where $\mathbf{n}=\nabla \varphi$ is a local normal vector to the interface pointing towards the liquid (cf. Sec. II A). However, we find that this approach leads to anisotropic artifacts in the free surface dynamics. Figure 1 documents an example.

Hence, for the present work, we take into account the interface orientation only at the contact line (corner nodes) where the boundary becomes inhomogeneous. As shown in Fig. 2, a contact line cell has links to solid wall (off-boundary, $\mathrm{S}$ ) nodes and to gas (off-boundary, G) nodes. Links to solid wall nodes (off-boundary nodes $\mathrm{S}$ ) are usually subject to a no-slip

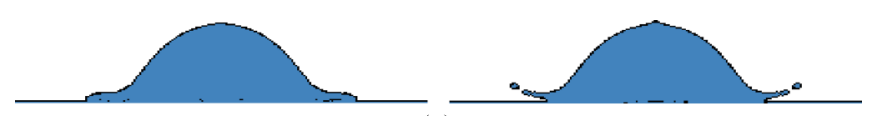

(a)

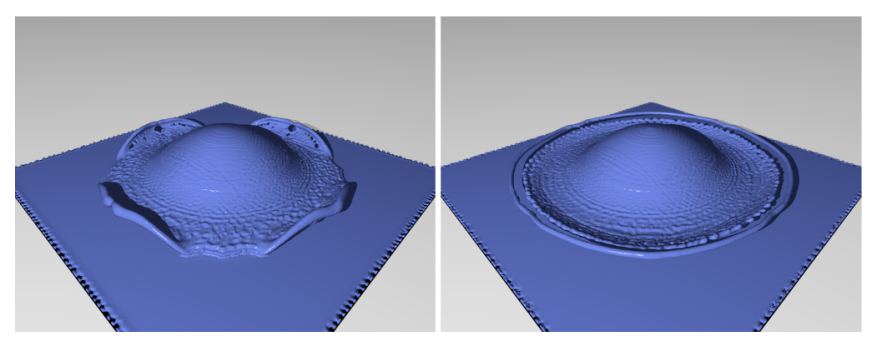

(b)

FIG. 1. Simulation of a droplet splashing onto a liquid film without surface tension. Reynolds number $\approx 250$. (a) Slice through domain $(x=y)$. Left: Imposing the free boundary condition on all links $\mathbf{c}_{q} \cdot \mathbf{n} \leqslant 0$ does not reproduce the rim correctly. Right: Rim clearly visible with the present implementation. (b) Isocontour of the tracked interface. Left: Imposing the free boundary condition on all links $\mathbf{c}_{q} \cdot \mathbf{n} \leqslant 0$ leads to incorrect crown formation. Right: Same simulation based on present implementation.

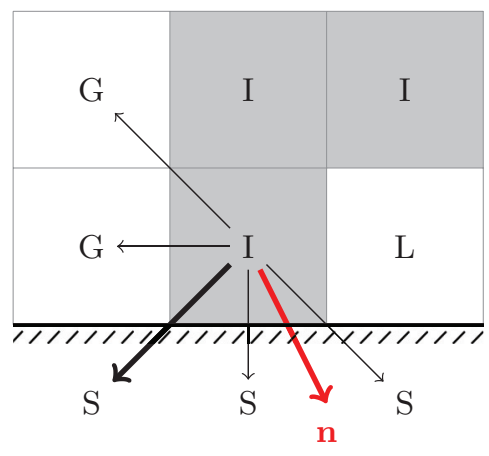

Node types:

L: liquid

I: interface

G: gas (off-boundary)

$\mathrm{S}$ : solid (off-boundary)

FIG. 2. Inhomogeneous boundary at an interface corner node with boundary-intersecting lattice directions indicated by arrows. The free boundary rule is imposed on the bottom-left direction (thick arrow) due to its orientation with respect to the interface normal $\mathbf{n}$.

condition. ${ }^{1}$ The present implementation replaces the no-slip condition with the free surface condition in corner nodes if the outgoing lattice direction has negative orientation with respect to the inward-oriented interface normal $\mathbf{n}$ projected into the solid wall.

The remaining part of this section lays out the three different approaches considered in this study to extract the local interface curvature $\kappa$ from the volume fraction $\varphi$. Notice that the notion of a lattice cell reflects the represented cubic volume with the length of one grid spacing and which is used to define the fill levels. However, the LBM data are more precisely located at lattice nodes which coincide with the centers of the cells.

\section{A. Finite-difference approximation}

As first proposed in Ref. [26], one way to approximate the curvature $\kappa$ of the boundary surface defined through the fill levels is to compute a finite-difference approximation to

$$
\kappa=-(\nabla \cdot \hat{\mathbf{n}}),
$$

where $\hat{\mathbf{n}}$ is the normalized gradient of the indicator function.

To obtain this gradient, we use central finite differences to approximate

$$
\mathbf{n}=\nabla \varphi,
$$

which can be interpreted also as a local normal vector to the free surface. We use the finite-difference approximation suggested in Ref. [42]. However, the curvature computation according to Eq. (7) means that second-order derivatives of the nonsmooth indicator function $\varphi$ are approximated by finite differences, which inevitably introduces larger errors. Hence, much work has been published (cf. for instance Ref. [43]) on effective ways to mollify the fill level information and smooth out surface tension in the "continuum surface tension" approach. We use the $K_{8}$ kernel with support radius $\epsilon=2.0$ (cf. Ref. [43]), i.e., only the next-neighbor information is included in the convolution.

\footnotetext{
${ }^{1}$ We use the bounce-back rule to realize no-slip boundary conditions, which assumes the wall position halfway between cell centers.
} 
Wetting properties are included by directly specifying an ideal equilibrium contact angle $\theta_{\mathrm{eq}}$ for solid boundaries. For obstacle cells with surface normal $\hat{\mathbf{n}}_{w}$ the boundary condition at the solid wall is

$$
\hat{\mathbf{n}}=\hat{\mathbf{n}}_{w} \cos \theta_{\mathrm{eq}}+\hat{\mathbf{n}}_{t} \sin \theta_{\mathrm{eq}}
$$

where $\hat{\mathbf{n}}_{t}$ is a tangent vector to the wall and normal to the contact line [26]. Since the wall position rarely coincides with the lattice nodes, the boundary value according to Eq. (9) is extrapolated to the obstacle node. Hereby, the vector $\hat{\mathbf{n}}_{t}$ is computed by projection of the interface normal at the fluid boundary cell $\mathbf{x}_{b}$ onto the wall. The boundary condition for the fill level in the obstacle cells (affecting the interface normal $\mathbf{n}\left(\mathbf{x}_{b}\right)$ in the boundary cells) is a reflection condition for the $\varphi$ values. Here we generally compute the boundary value for the obstacle cells $\mathbf{x}_{o} \notin \Omega$ based on the neighboring inner nodes $\mathbf{x}_{o}+\mathbf{c}_{q} \in \Omega$ using the formula

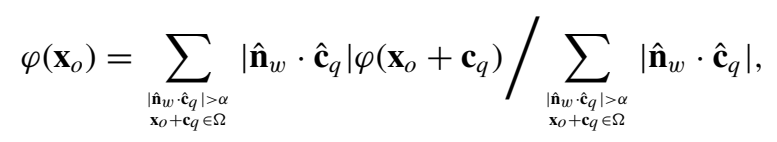

with an aperture $\alpha=\sqrt{2} / 2$, to achieve a smoothed reflection for the boundary values.

\section{B. Triangular reconstruction based on piecewise linear interface construction}

In Ref. [31], a curvature computation is suggested based on a local triangulation of interface points in a $3 \times 3 \times 3$ neighborhood around each interface cell. The interface points are determined using a piecewise linear interface construction (PLIC) approach [3]. For an interface cell centered around $\mathbf{x}_{i}$, let $P$ be the half-space $P=\left\{\mathbf{x} \mid(\mathbf{x}-\mathbf{p}) \cdot \mathbf{n}\left(\mathbf{x}_{i}\right) \geqslant 0\right\}$, where $\mathbf{p}=\mathbf{x}_{i}+a \mathbf{n}\left(\mathbf{x}_{i}\right)$ is a point within the corresponding unit volume $V\left(\mathbf{x}_{i}\right)$. Now the interface point $\mathbf{p}$ is defined such that the cut-off volume $V \cap P$ satisfies

$$
\operatorname{Vol}(V \cap P)=\varphi\left(\mathbf{x}_{i}\right) .
$$

We determine $a$ iteratively, similarly to Ref. [44], with an error bound of $\approx 4 \times 10^{-13}$ (40 iterations in a bisection algorithm) assuming an exact surface normal. The interface point can be computed in one step together with the estimation of the surface normals. For the latter we employ again a finite-difference scheme to Eq. (8), however, without any convolution step. Alternative, higher-order PLIC algorithms are discussed in Refs. [38,57].

Once the interface points are determined by the PLIC scheme, the algorithm described in Ref. [31] is used to construct a local "triangle fan" from the interface points within the local neighborhood. Then, a variant of the algorithm described in Ref. [45] determines the curvature of this polygonal surface. Notice that the described TR scheme as well as the curvature estimation by LSQR of Sec. II C are based solely on the surface points and use the gradient information represented by the interface normals only as far as it is needed to construct these interface points. The TR method can be extended to support adhesive boundary conditions. In Ref. [46], a way to extend the local triangulations at solid boundaries to achieve an "artificial curvature" matching with a desired equilibrium contact angle is described. However, the implementation of the geometric construction is difficult in three dimensions. Also, we found the results obtained from that method often not convincing, which motivated the least-squares-based approach of the following section.

\section{Least-squares reconstruction based on piecewise linear interface construction}

The third approach to include surface tension consists in reconstructing the interface as a quadratic function in each $3 \times$ $3 \times 3$ neighborhood around an interface cell. It has previously been described in Ref. [32], however, without the inclusion of wall adhesion effects. Like the TR approach, it is based on the PLIC of interface points described above in Sec. II B. Let $\hat{\mathbf{t}}_{u}$, $\hat{\mathbf{t}}_{v}$, and $\hat{\mathbf{n}}$ be a local orthonormal basis, i.e., $\hat{\mathbf{t}}_{u} \hat{\mathbf{t}}_{v}$ tangential to the interface, and $\mathbf{p}$ the local interface point. Now assume that $i$ is indexing all the remaining interface cells in a $3 \times 3 \times 3$ neighborhood. The interface cell data $\left(\hat{\mathbf{n}}_{i}, \mathbf{p}_{i}\right)$ is used to fit the model function

$$
f(u, v)=A u^{2}+B v^{2}+C u v+H u+I v+J
$$

with parameters $(A, B, C, H, I, J)$, by minimizing the error

$$
E=\sum_{i}\left|f\left(u_{i}, v_{i}\right)-f_{i}\right|^{2}
$$

Here $u_{i}=\left(\mathbf{p}_{i}-\mathbf{p}\right) \cdot \hat{\mathbf{t}}_{u}, v_{i}=\left(\mathbf{p}_{i}-\mathbf{p}\right) \cdot \hat{\mathbf{t}}_{v}$, and $f_{i}=\left(\mathbf{p}_{i}-\mathbf{p}\right)$. $\hat{\mathbf{n}}$. This yields a linear least-squares problem that has to be solved locally. We obtain the best results when fixing the constant parameter $J=0$, i.e., accepting only solutions that interpolate the surface point $\mathbf{p}$ of the respective interface cell. We use the implementation from the LAPACK library [47] based on $\mathrm{QR}$ decomposition of the corresponding system matrix. Once $f$ is determined the curvature can be evaluated analytically, using

$$
\kappa=\frac{A\left(1+I^{2}\right)+B\left(1+H^{2}\right)-2 C H I}{\left(\sqrt{1+H^{2}+I^{2}}\right)^{3}} .
$$

The approach can be seen as a modified version of the parabolic reconstruction of surface tension (PROST) scheme from Ref. [48]. Both schemes fit a parabolic function in a local neighborhood around each interface cell. However, as a major difference, PROST fits $f(x, y, z)$ directly to the fill levels minimizing the error $\sum_{i}\left(V_{i}(f)-\varphi_{i}\right)^{2}$ of the cut-off volumes $V_{i}$ that the isosurface $f(x, y, z)=0.5$ cuts out of the interface cell $i$. This makes the least-squares problem nonlinear and $f$ has to be determined by iteratively computing the error and updating of coefficients. The scheme described here is computationally less expensive.

To include the effect of boundary adhesion, we extend the method in the following way: If the local $3 \times 3 \times 3$ neighborhood contains an obstacle cell, then for each contactline cell (i.e., an interface cell that has an obstacle cell as neighbor) from the same neighborhood, one contact point is approximated with a contact normal $\hat{\mathbf{m}}$ defined according to Eq. (9). In the contact point, we require

$$
\nabla f\left(u_{c}, v_{c}\right)=-\left(m_{u}, m_{v}\right) / m_{n},
$$

where $m_{u}=\hat{\mathbf{m}} \cdot \hat{\mathbf{t}}_{u}, m_{v}=\hat{\mathbf{m}} \cdot \hat{\mathbf{t}}_{v}$, and $m_{n}=\hat{\mathbf{m}} \cdot \hat{\mathbf{n}}_{u}$, and $u_{c}, v_{c}$ are the coordinates of the contact point in the locally defined 


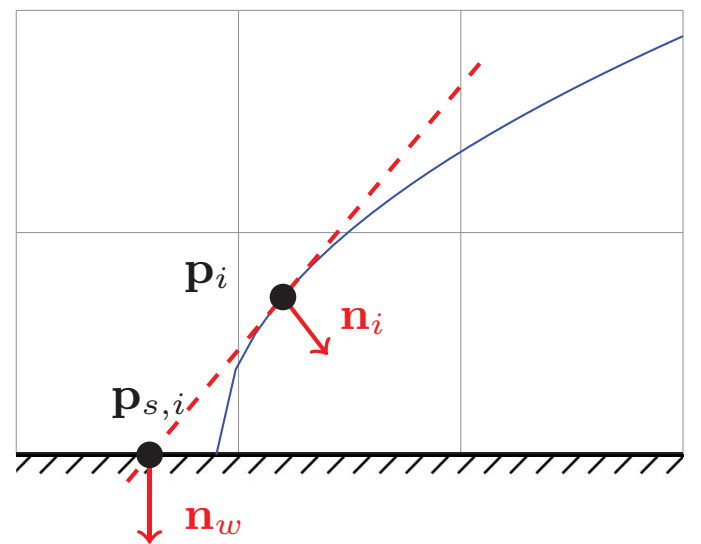

FIG. 3. Determination of contact point $\mathbf{p}_{s, i}$ for a contact line cell. The PLIC segment (dashed line) defined by interface point $\mathbf{p}_{i}$ and interface normal $\mathbf{n}_{i}$ is extended and intersected with the obstacle wall.

tangential plane. If the neighboring interface cell is the center of the current $3 \times 3 \times 3$ neighborhood, then we use Eq. (14) as a constraint to the respective optimization problem given by Eqs. (12a) and (12b). Otherwise, the condition is simply included in the optimization of the error, Eq. (12b).

To obtain the contact point, we construct the closest intersection of the interface segment of the contact line cell with the solid surface as in Fig. 3.

\section{Current limitations}

The interface tracking of the present implementation defines interface cells as active lattice Boltzmann cells that have a D3Q19 neighborhood containing both gas and liquid cells. This definition turned out to impose a limitation when simulating thin liquid films on wetting surfaces and with contact angles below $45^{\circ}$. As shown in Fig. 4, the thickness of a liquid film on solid substrate has to be resolved at least by one liquid cell in height for the method to work correctly. A film thickness smaller than 1 lattice unit is not supported. For strongly wetting surfaces $\left(\theta_{\mathrm{eq}}<45^{\circ}\right)$, we often observe anisotropic errors because it is then problematic to impose

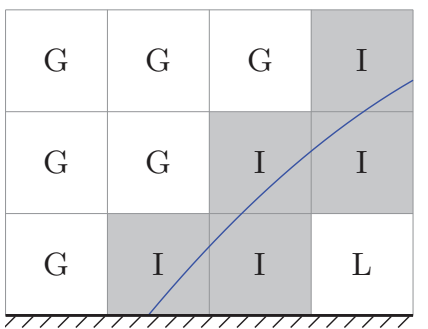

(a)

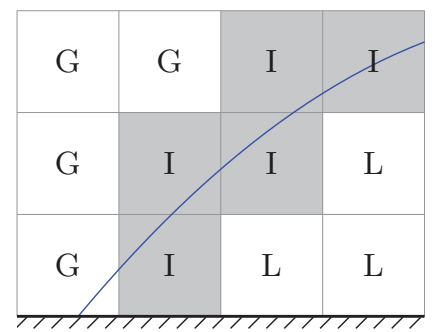

(b)
FIG. 4. The bottom-left interface (I) cell in (a) has several gas (G) neighbors but no liquid neighbor (L). Hence, the configuration of (a) is not supported by the present implementation. Shown in (b) is a valid configuration since all interface cells have both liquid and gas neighbors. The minimum supported film thickness is therefore at least one (liquid) lattice cell. (a) Invalid; (b) valid. the correct contact angles according to the LSQR method (Sec. IIC) and the TR method (Sec. IIB): Depending on the approximated interface position represented by the fill level information, the computed curvature values then tend to oscillate and overshoot. In Fig. 4(b), for instance, the approximation of the interface as a smooth surface through the two leftmost interface cells can be expected to be erroneous under the condition of an acute intersection angle $\left(\theta_{\mathrm{eq}}<45^{\circ}\right)$ with the solid surface. It is important to notice that these restrictions are specific to the presented FSLBM algorithm, while the presented curvature reconstruction schemes can be applied in any VOF context.

\section{NUMERICAL RESULTS}

The numerical results presented in the following have been obtained with the waLBerla lattice Boltzmann framework [49] that includes the FSLBM implementation described in [31].

\section{A. Equilibrium spherical bubble}

The standard benchmark for surface tension models is a static equilibrium bubble. If the curvature estimation would return the exact value $\kappa=1 / R$ everywhere on the interface, then the solution to the problem would be a perfectly vanishing velocity field. Due to the existing errors, however, spurious currents occur around the interface from regions with overestimated Laplace pressure to positions underestimating the value (cf. Fig. 5) [3]. For nonsophisticated methods, the magnitude of the spurious velocities can be related to the ratio $\sigma / \mu$, of surface tension and dynamic viscosity $\mu=\rho \nu$, with a constant prefactor $\leqslant 1$. This means that the error is

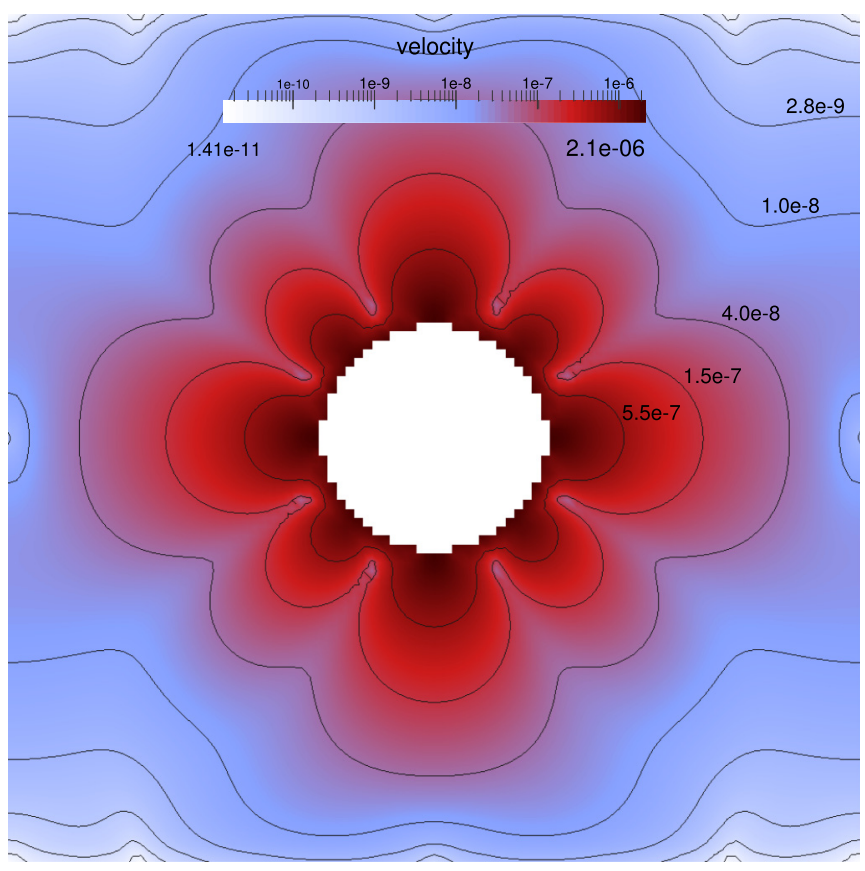

FIG. 5. Slice through a domain containing a single gas bubble. The color indicates the magnitude (lattice units) of the spurious currents due to errors in the curvature estimation with the FD approach. 
independent of the spatial resolution, or converging very slowly, and thus poses a limitation in terms of applicability to problems involving dominant capillary forces. See Refs. $[28,43,50]$ for a discussion of the problem in connection with the VOF method.

Here we adopt the problem stated in Ref. [48] using nondimensional values with respect to a cubic domain of unit length, containing a spherical bubble of radius $R=$ 0.125 centered at $(0.5,0.5,0.5)$. We apply no-slip boundary conditions at the top $(z=1)$ and bottom $z=0$ sides of the domain and periodicity along all other directions. The viscosity of the liquid of density $\rho=4$ is set to $\mu=1$, and the surface tension parameter is $\sigma=0.357$. The dimensionless Ohnesorge number $(\mathrm{Oh}=\mu / \sqrt{\sigma \rho R})$ corresponding to the given problem is $\mathrm{Oh} \approx 2.37$. Notice that in Ref. [48] the bubble is actually a second fluid of the same density and viscosity as the liquid (two phase), while in our case the flow inside the gas bubble is not simulated but represented only in terms of a gas pressure value that is dynamically adjusted according to the changes of the gas volume upon interface advection (free surface flow with bubble model [51,52]). We remark that the present test case is appropriate to evaluate the error in the curvature computation only. Since there is no flow velocity in this test case aside from the spurious currents, one expects no significant error contribution from the advection of the indicator function or the LBM. We have therefore evaluated the test case first in the standard case with a static frame of reference (Sec. III A 1) and second in a moving frame of reference (Sec. III A 2) with a constant uniform background velocity added to the flow.

\section{Static frame of reference}

We perform a resolution study varying the grid spacing between the values $\delta_{x}=1 / 48,1 / 96,1 / 144,1 / 192$ at a fixed time step of $\delta_{t}=10^{-4}$, which yields the resolutiondependent lattice relaxation times $\tau=0.6728,1.191,2.055$, and 3.264. The surface tension parameter in lattice units varies accordingly and takes the values $\sigma_{L} / 10^{-3}=0.0987$, $0.7896,2.665$, and 6.317 . For initialization of the fill levels at $t=0$ with the spherical geometry, we employ a spatial subdivision technique, refining each discrete cell volume by a factor of 100 along each coordinate. The simulation then exhibits a series of pressure disturbances until the numerical equilibrium is reached. Figure 6 shows the development of the maximal and average flow velocity within the domain for 500000 time steps. After a certain number of time steps the shock wave is sufficiently decayed for both the TR and the LSQR method, such that both maximal and average flow velocity within the domain become smaller than $\lesssim 10^{-10}$ in magnitude. The FD approach, however, does not converge and enters into an oscillating behavior instead. Here the spurious currents are large enough to trigger changes in the layer of interface nodes. This also introduces sudden changes in the curvature computation, thus explaining the oscillations. Figure 7 shows the maximal and average velocity within the domain for different spatial resolutions and various methods. The strength of the spurious currents obtained with the FD method are in accordance with the values reported in Ref. [48], where a similar approach (CSF) is used for referencing. The curvature information obtained by geometric reconstruction

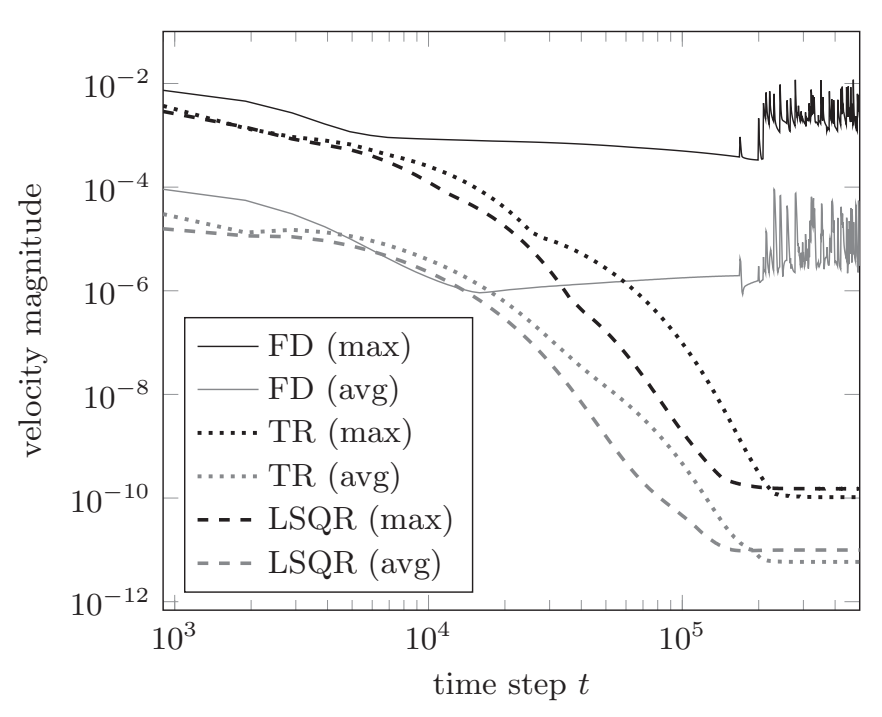

FIG. 6. Temporal evolution of maximal and average (spurious) velocity for the different surface tension models at a fixed grid spacing $\delta_{x}=1 / 96$. After the decay of an initial shock, the magnitude of the spurious currents in the system can be evaluated. The FD model shows the largest errors and often leads to oscillative behavior. Similar behavior is obtained for $\delta_{x}=1 / 48,1 / 144$, and $1 / 192$.

(TR and LSQR methods) is much more accurate than the FD approximation and reduces the spurious velocities almost down to the order of machine precision.

We also evaluate the accuracy of the curvature values obtained for the numerical equilibrium, i.e., the state reached after 500000 time steps. Figure 8(a) compares the error in curvature at various grid spacings for the three different methods. Only the plot for the LSQR method indicates a second-order rate of convergence. However, it is clear that the occurrence of spurious currents is not due to constant over- or underestimation of curvature but rather because of its variance with the node position. This becomes obvious when compared to Fig. 8(b), which shows the standard deviation over all interface nodes in the final state to the resulting spurious currents of Fig. 7.

\section{Moving frame of reference}

When dealing with dynamical problems numerical errors in the advection of the indicator function $\varphi$ are often critical. This holds in particular for the present test case, since any errors in the fill levels will introduce an additional error into the computed curvature values. To study effects of advection, we add a constant background velocity of $\mathbf{u}_{0}=(1,0,0)^{T}$ to the test case. This means that there is now a constant advection involved and the spurious currents appear as a deviation from the background velocity. For $\delta_{x}=1 / 96, \delta_{t}=2.5 \times 10^{-5}$ (lattice relaxation time $\tau=0.6728, u_{x}=0.0024 \delta_{x} / \delta_{t}$ ), we run the simulation for $T=L / u_{0, x}$ time steps, i.e., the bubble traverses the periodic domain of length $L$ exactly one time. This can be interpreted as a moving frame of reference while, physically, the setup is equivalent to the static version of Sec. III A 1. Measuring the curvature error over time, Fig. 9 exhibits a dramatic increase in error as compared to the static test case. The errors of the reconstructive methods, 


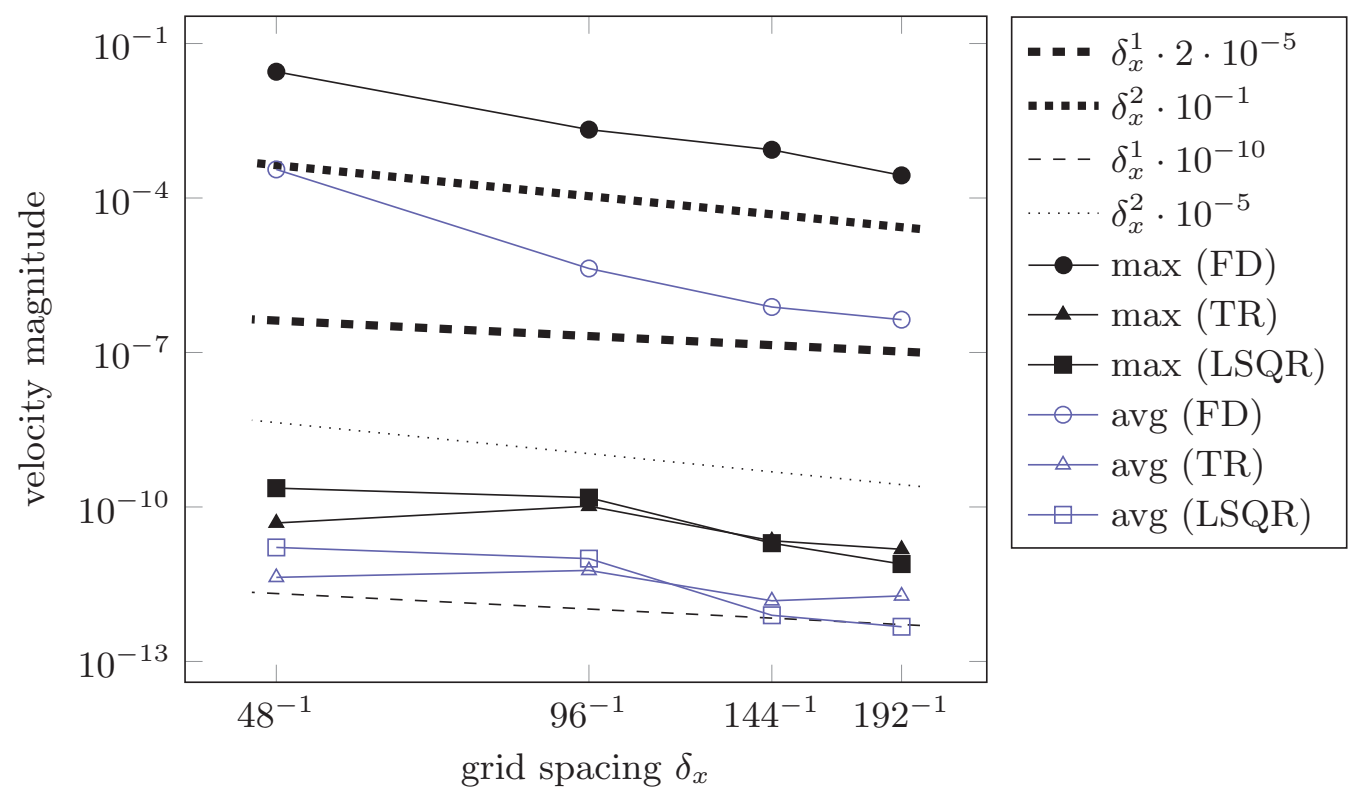

FIG. 7. Dependency of spurious velocity (maximum and average) on grid spacing $\delta_{x}$ for the three different models evaluated after 500000 time steps. The dotted and dashed lines represent first- and second-order slopes, respectively. For the FD scheme, the plot indicates a first-order convergence for both maximum and average spurious flow speed. The errors of the FD scheme are orders of magnitude above those of the methods based on surface reconstruction.

LSQR and TR, are now significantly larger than the error of the FD model. A possible reason is that the FD model is more diffusive than the reconstruction methods and thus less sensitive to errors in the indicator function field. A grid study with spatial steps $\delta_{x}=1 / 48,1 / 96,1 / 144,1 / 192$ and corresponding time steps $\delta_{t}=1 \times 10^{-4}, 2.5 \times 10^{-5}, 1.11 \times$ $10^{-5}, 6.25 \times 10^{-6}$ (diffusive scaling) revealed that these errors do not converge with the grid spacing. Figure 10 shows that the shape of the bubble after advection deviates notably from a true sphere. In accordance with the increased errors in curvature, the reconstruction based schemes show the most deviation. Also, in this case the spurious currents no longer converge for either method. This indicates that there is an additional error that stems from the advection of the indicator function $\varphi$.

Since we are not aware of any numerical evaluation of the FSLBM advection scheme, we supplement the Laplace test with a convergence check of the indicator function values. To this end, a gas bubble of diameter $d=10 \delta_{x}$ is placed inside a periodic computational domain $\Gamma$ with a prescribed uniform velocity $\mathbf{u}_{0}=(0.05,0,0)^{T} \delta_{x} / \delta_{t}$. The LBM

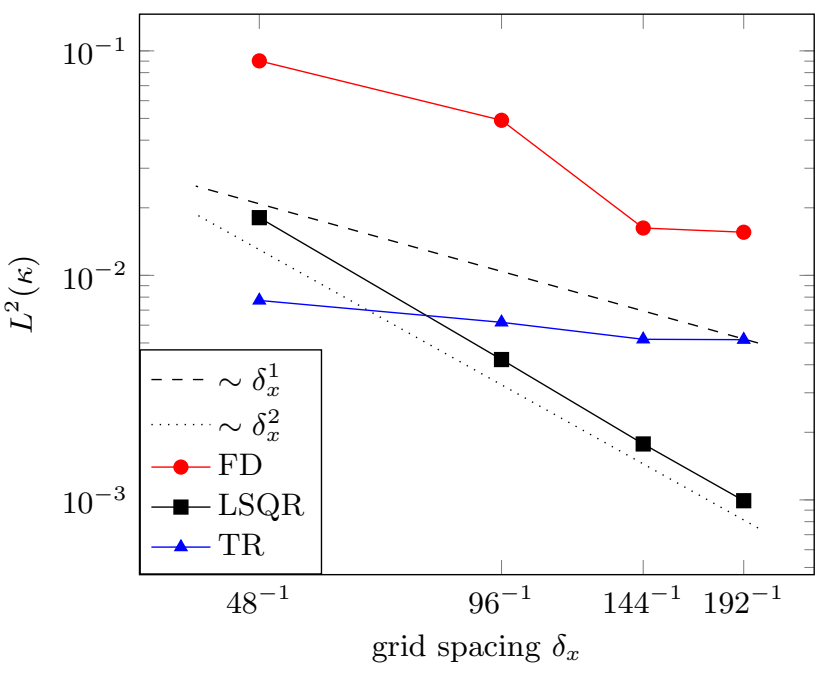

(a)

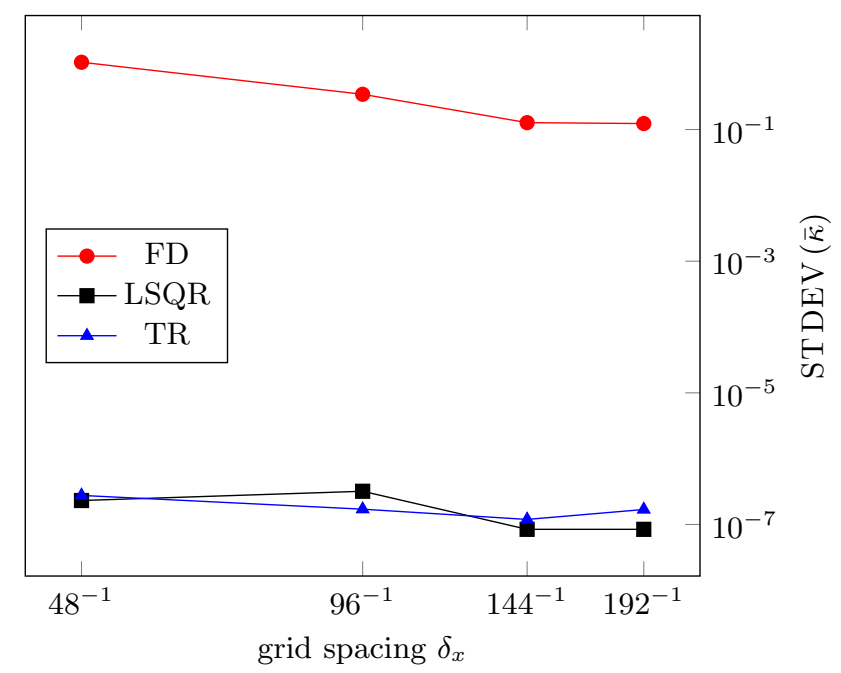

(b)

FIG. 8. Comparison of curvature estimation for a stationary bubble after 500000 time steps. For the FD model, the included graphs are somewhat arbitrary, because the values oscillate over time, analogously to the spurious currents in cf. Fig. 6. (a) The $L^{2}$ norm of the curvature error. Only the LSQR curvature error does converge in the test, with a rate of convergence in $\mathcal{O}\left(\delta_{x}^{2}\right)$. (b) Standard deviation of average curvature values. The FD scheme has the largest standard deviation. Consecutively, the FD scheme generates the largest spurious currents. 


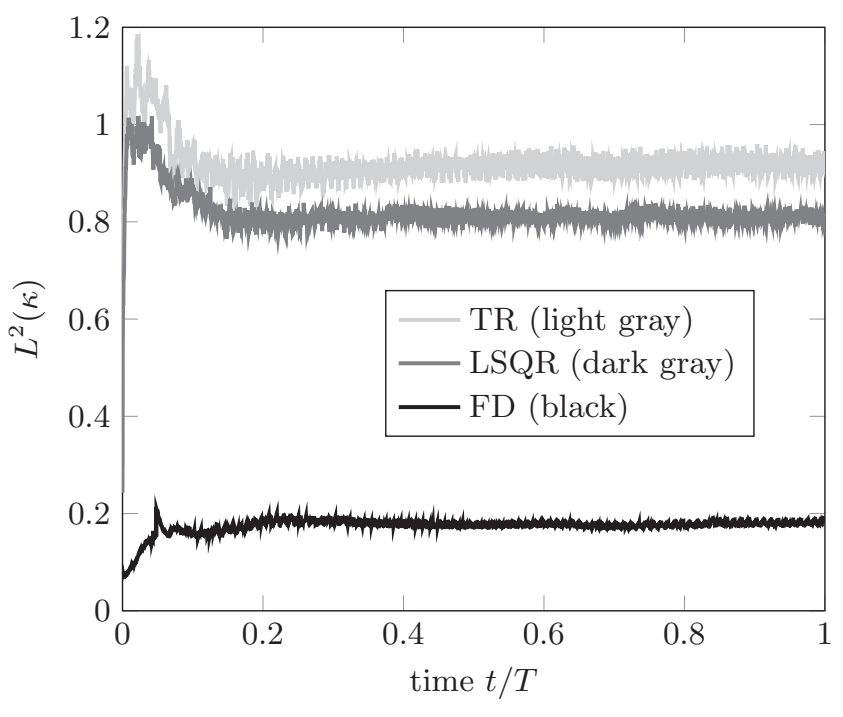

FIG. 9. $L^{2}$ error in curvature for the three different surface tension models for a moving frame of reference. Simulation of a spherical bubble during traversal a periodic domain with uniform velocity. At $t=0$ the bubble is initialized to a nearly ideal (spherical) shape. Due to errors in the advection operator and the surface tension models the error increases and after $t=0.4$ oscillates around a fixed value. The FD scheme is less sensitive to errors in the fill levels, presumably because it is more diffusive and based on the mollified indicator function.

data are thus constant with $\mathbf{f}(\mathbf{x}, t)=\mathbf{f}^{\mathbf{e q}}\left(p_{g} / c_{s}^{2}, \mathbf{u}_{\mathbf{0}}\right)$, where $p_{g}$ is the constant reference pressure. This excludes any error contribution by the LBM or the surface tension modeling to the indicator function $\varphi$. From the prescribed LBM data we compute the advection of the bubble in terms of the indicator function according to Eqs. (3a) and (3b). After a time $T=d / u_{0, x}$ the bubble has moved a distance equal to its diameter. To evaluate the error, we use the $L^{1}$ and $L^{2}$ error norms by comparing the fill levels at time $T$ to the initial configuration $t=0$, i.e.,

$$
\begin{aligned}
L^{1}(\varphi) & =\frac{\sum_{\mathbf{x} \in \Gamma}\left|\varphi\left(\mathbf{x}+T \mathbf{u}_{0}, T\right)-\varphi(\mathbf{x}, 0)\right|}{\sum_{\mathbf{x} \in \Gamma}|\varphi(\mathbf{x}, 0)|}, \\
L^{2}(\varphi) & =\sqrt{\frac{\sum_{\mathbf{x} \in \Gamma}\left[\varphi\left(\mathbf{x}+T \mathbf{u}_{0}, T\right)-\varphi(\mathbf{x}, 0)\right]^{2}}{\sum_{\mathbf{x} \in \Gamma} \varphi(\mathbf{x}, 0)^{2}}} .
\end{aligned}
$$

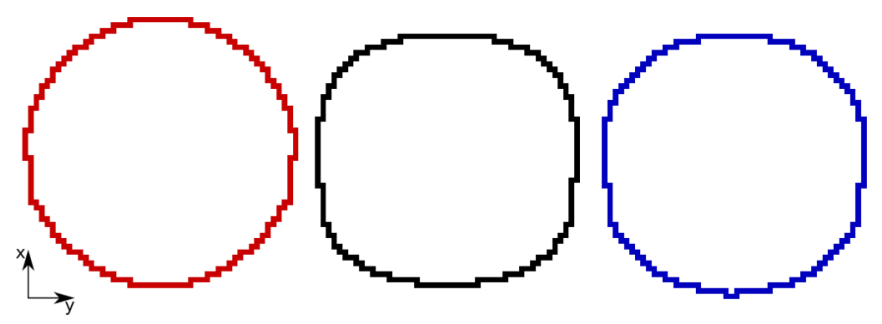

FIG. 10. Comparison of the shapes of the bubbles after advection along $x$ axis with surface tension. From left to right: FD, LSQR, TR. Visualization shows the layer of interface cells in the $x-y$ plane through the center of the bubbles at time $0.94 T$ (150 000 time steps). The initial radius of the bubble was $24 \delta_{x}$.
TABLE I. $L^{1}$ and $L^{2}$ errors in the indicator function $\varphi$ due to advection. The translation of a spherical bubble of diameter $d$ over a distance $d$ in uniform velocity field (constant velocity $\mathbf{u}_{0}$ along $x$ axis) is evaluated for different time and grid spacings $\delta_{t}$ and $\delta_{x}$. The test

\begin{tabular}{|c|c|c|c|c|c|c|c|}
\hline \multirow[b]{2}{*}{$d / \delta_{x}$} & \multirow[b]{2}{*}{$\delta_{x}$} & \multicolumn{3}{|c|}{ Convective } & \multicolumn{3}{|c|}{ Diffusive } \\
\hline & & $\delta_{t}$ & $u_{0, x} \delta_{t} / \delta_{x}$ & $L^{1}(\varphi) L^{2}(\varphi)$ & $\delta_{t}$ & $u_{0, x} \delta_{t} / \delta_{x}$ & $L^{1}(\varphi) L^{2}(\varphi)$ \\
\hline 10 & 1 & 1 & 0.05 & $3.00 \% 9.38 \%$ & 1 & 0.05 & $3.00 \% 9.38 \%$ \\
\hline 20 & $1 / 2$ & $1 / 2$ & 0.05 & $1.63 \% 7.19 \%$ & $1 / 4$ & 0.025 & $1.38 \% 6.17 \%$ \\
\hline 40 & $1 / 4$ & $1 / 4$ & 0.05 & $1.25 \% 7.38 \%$ & $1 / 16$ & 0.0125 & $0.85 \% 5.39 \%$ \\
\hline 80 & $1 / 8$ & $1 / 8$ & 0.05 & $1.17 \% 8.44 \%$ & $1 / 64$ & 0.00625 & $0.68 \% 5.87 \%$ \\
\hline
\end{tabular}
case has been performed for both convective and diffusive scaling.

The test case is repeated with successively refined grid spacing, under convective scaling $\left(\delta_{t} \sim \delta_{x}\right)$ and diffusive scaling $\left(\delta_{t} \sim \delta_{x}^{2}\right)$. The exact parametrizations and the resulting errors are collected in Table I. Figure 11 shows dependency of the respective errors on the lattice resolution. The indicated convergence rate is below first order, independent of the used scaling and error norm. This means that one cannot assert first-order convergence with the present advection scheme in a simple translation test.

With respect to the moving Laplace bubble test, the increased error observed in Fig. 9 as compared to the static one presented in Sec. III A 1, as well as the degenerated bubble shapes after advection (cf. Fig. 10) suggests the following explanation. Any errors in the indicator function affect also the curvature computation, which explains the temporal oscillation of the error as the bubble moves relative to the grid (cf. Fig. 9). The reconstruction methods (TR and LSQR) seem more sensitive to the advective errors than the simpler FD scheme and hence are less stable in the dynamic case. Even though the curvature estimates of LSQR and TR are more accurate and effectively reduce spurious currents in

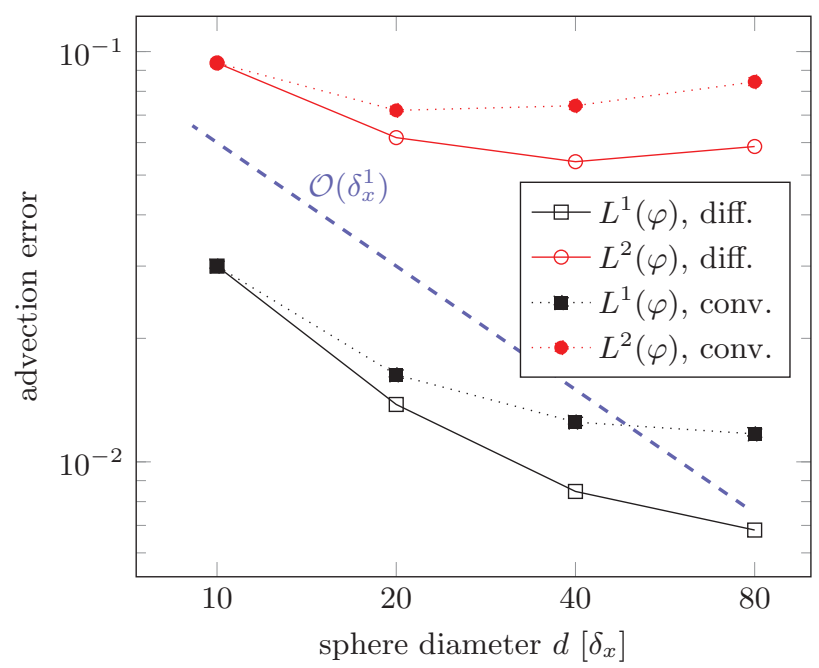

FIG. 11. Dependency of $L^{1}$ and $L^{2}$ errors in the indicator function $\varphi$ on lattice resolution under convective and diffusive scaling (cf. Table I). Test case consisted of a spherical bubble of diameter $d$ is advected over a distance $d$. The indicated order of convergence is below 1 . 


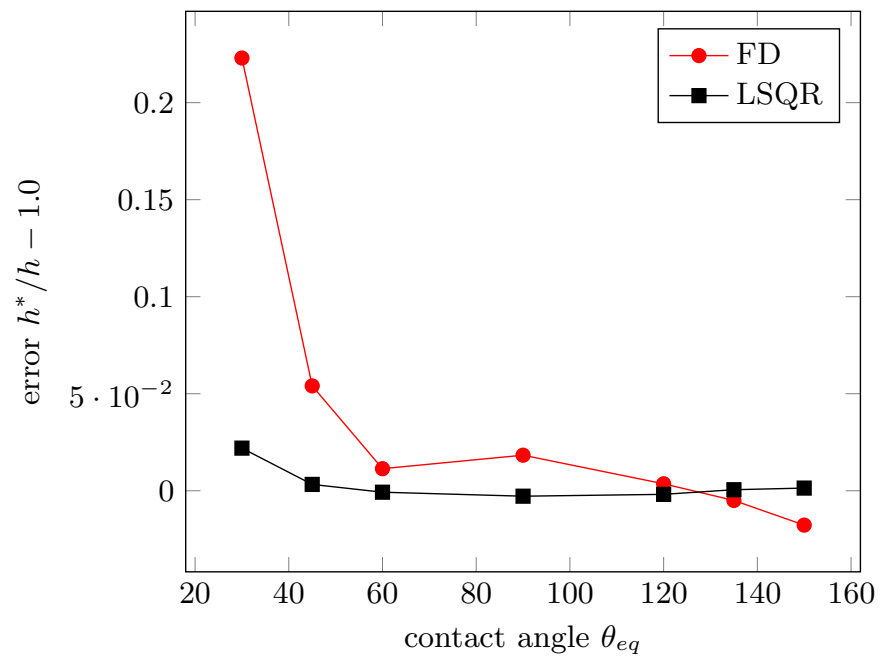

(a)

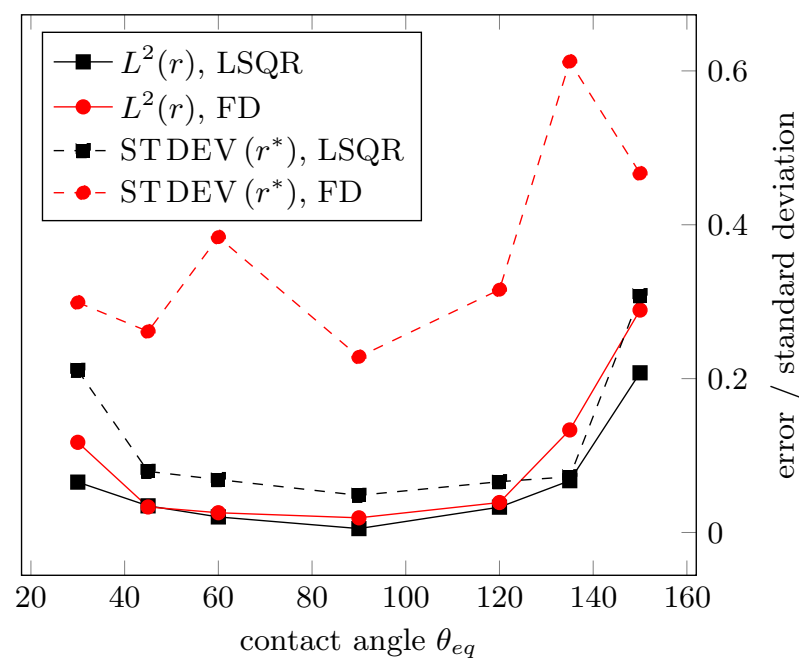

(b)

FIG. 12. Sessile droplet equilibrium on solid surface for various equilibrium contact angles $\theta_{\mathrm{eq}}$ simulated with grid spacing $\delta_{x}=1 / 96$. Deviation of numerical equilibrium from ideal equilibrium for FD and LSQR scheme. (a) The relative deviation of the simulated droplet height $h^{*}$. (b) The relative deviation of contact line radius in the $L^{2}$ norm and the standard deviation of $r^{*}$ computed over all contact line cells as a measure for anisotropic artifacts.

the static benchmarks, the combination with the low-order advection scheme is problematic.

\section{B. Droplets on wetting boundaries}

\section{Equilibrium sessile droplets}

Next, we evaluate the error at the contact line with solid boundaries. We change the setup of Sec. III A to a spherical cap shaped droplet, such that the initial state of the droplet is close to the ideal equilibrium. The equilibrium is a spherical cap resting on the wall, with a sphere radius $R$ related to the equilibrium contact angle $\theta_{\mathrm{eq}}$ of the wall by $R=h /\left(1-\cos \theta_{\mathrm{eq}}\right)$. Given the volume of the droplet $V$, the ideal equilibrium height of the droplet is

$$
h=\sqrt[3]{\frac{V}{\pi\left(\frac{1}{1-\cos \theta_{\mathrm{eq}}}-\frac{1}{3}\right)},}
$$

and the ideal contact line radius $r$ (base radius of the spherical cap) is

$$
r=\sqrt{\frac{1}{3}\left(\frac{6 V}{\pi h}-h^{2}\right)} .
$$

The simulated height $h^{*}$ and contact line radius $r^{*}$ have to be approximated from the indicator function, as

$$
h^{*}=\max _{\mathbf{x} \in I}\left[x_{z}+\varphi(\mathbf{x})-0.5\right]
$$

and

$$
r^{*}=\frac{1}{\left|I_{c}\right|} \sum_{\mathbf{x} \in I_{c}} \tilde{r}(\mathbf{x}),
$$

where $I$ denotes the set of interface nodes and $I \supset I_{c}$ the set of contact line nodes. Hereby, $\tilde{r}$ is the local approximation

$$
\tilde{r}(\mathbf{x})=\left\|\mathbf{x}-\mathbf{x}_{o}\right\|+[\varphi(\mathbf{x})-0.5],
$$

where $\mathbf{x}_{o}$ is the ideal center of the circular contact line. This approximation reflects that the nodes $\mathbf{x} \in I$ are the centers of the corresponding interface cells. Furthermore, the error in the contact line is evaluated using the $L^{2}$ error definition,

$$
L^{2}(r)=\sqrt{\frac{\sum_{\mathbf{x} \in I_{c}}[r-\tilde{r}(\mathbf{x})]^{2}}{\sum_{\mathbf{x} \in I_{c}} r^{2}}} .
$$

Error analysis. The error analysis involved the ideal contact angles $\theta_{\mathrm{eq}}=30^{\circ}, 45^{\circ}, 60^{\circ}, 90^{\circ}, 120^{\circ}, 135^{\circ}$, and $150^{\circ}$ at a constant resolution with $\delta_{x}=1 / 96$ and $\delta_{t}=10^{-4}$. In all cases, the droplet volume $V$ was chosen equal to that of a hemisphere of radius $R=0.125$. Figure 12 shows the relative errors in height of the droplet shape obtained in the simulations, and the relative $L^{2}$ error in the simulated contact line radius. For the most extreme contact angles $\theta_{\text {eq }}$, the droplet height and contact line move away significantly from the initial (ideal) equilibrium position, increasing the respective errors until the numerical equilibrium is reached. Figure 12(b) also shows the standard deviation in the measured contact line radius, computed over all contact line cells. While the errors in the contact line seem to be comparable in size, the higher values in $\operatorname{STDEV}\left(r^{*}\right)$ obtained with the FD scheme indicate that the simulated contact lines are more anisotropic than with the LSQR scheme.

Convergence study. A convergence study was performed for the selected equilibrium positions of $\theta_{\mathrm{eq}}=60^{\circ}, 90^{\circ}$, and $120^{\circ}$ by altering the grid spacing to $\delta_{x}=1 / 48,1 / 96$, and $1 / 144$ using diffusive scaling for the time step. Figure 13 compares the $L^{2}$ errors obtained by the FD and the LSQR schemes and shows that the LSQR error is generally smaller. The error behavior in terms of grid dependency is somewhat irregular for both schemes. However, at least for the LSQR model, the convergence rate of the error appears to be approximately first order. Since the construction of the contact points described in Sec. II C exploits the linear approximation 


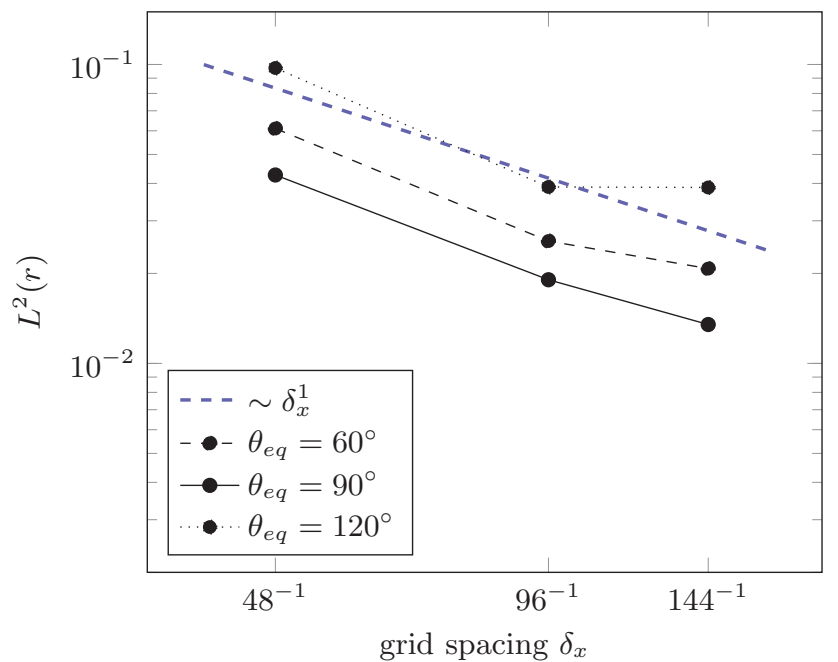

(a)

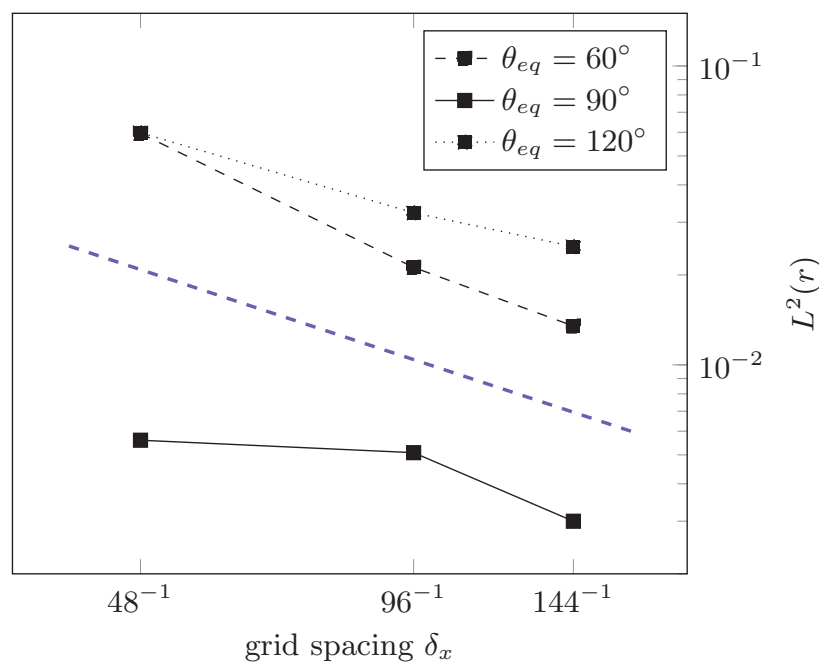

(b)

FIG. 13. Grid convergence study of the $L^{2}$ error in the simulated contact line radius; the FD approach and LSQR approach in comparison. (a) FD scheme. (b) LSQR scheme.

of the reconstructed interface, the observed first-order error is in accordance with the expected behavior.

\section{Droplet spreading on wetting boundaries}

Because of the limitations described in Sec. IID, for extreme contact angles, $\theta_{\mathrm{eq}}<45^{\circ}$ or $\theta_{\mathrm{eq}}>135^{\circ}$, we focus our study to a few dynamical cases. For the inertial regime (low Ohnesorge number), a spherical droplet in contact with an adhesive plane substrate will start to spread according to the power law

$$
r(t) \sim t^{0.5}
$$

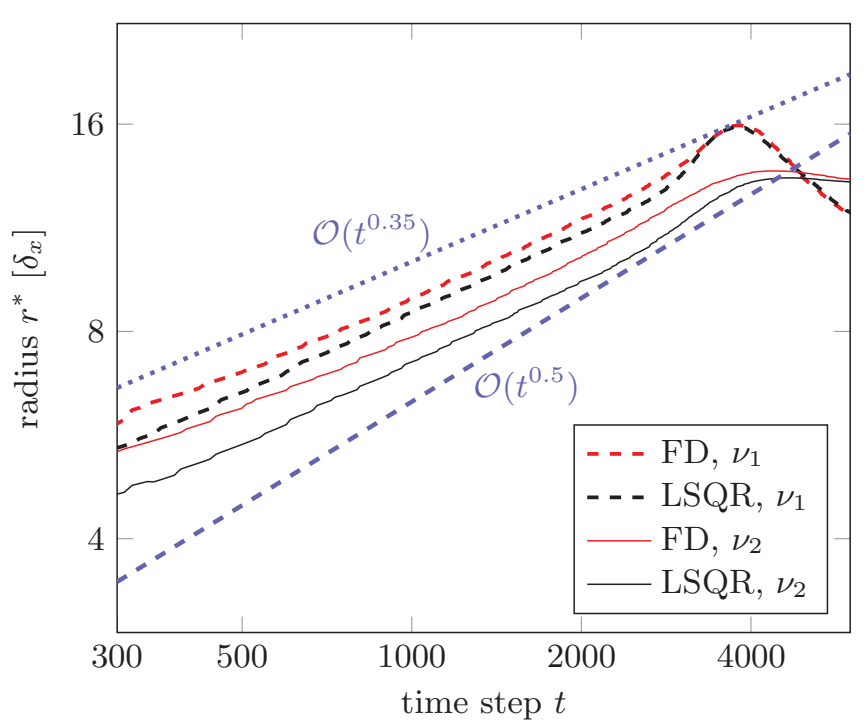

FIG. 14. Contact line dynamics of the spreading droplet; comparison of simulations based on the FD scheme and the LSQR scheme. The test case is repeated for two different viscosities, $v_{1}=3.32226 \times 10^{-3}$ and $v_{1}<v_{2}=1.66113 \times 10^{-2}$ (lattice units). Both schemes tend to underestimate the ideal spreading law of $r(t) \sim t^{0.5}$. where $r$ is the radius of the circular contact line [53]. A numerical simulation of contact line dynamics requires the accurate modeling of a slip condition to resolve the stress singularities in the moving contact line. Furthermore, it is in general not sufficient to work with a static contact angle model that imposes the equilibrium contact angle $\theta_{\mathrm{eq}}$ everywhere at the contact line [54]. In the presented FSLBM, the interface representation by volume fractions introduces a certain amount of numerical slip that allows the free surface to move in the contact line [55]. This numerical slip is related to the grid spacing and does not necessarily recapture the correct physics. We have not introduced a dynamic contact angle model.

We simulate the spreading of a droplet of radius $R=10$ lattice units on a flat plate with ideal equilibrium contact angle $\theta_{\text {eq }}=85^{\circ}$. The droplet is initialized as a sphere placed at a distance of $R$ from the solid boundary. Due to a discretization effect, the simulated initial contact line has a positive radius $r>0$, such that the adhesive boundary condition can be imposed in the contact line cells. The surface tension constant is chosen $\sigma=4.3189 \times 10^{4}$ for a fluid of lattice reference density $\rho=1.0$ and kinematic viscosity $v$ of $v_{1}=3.32226 \times$ $10^{-3}$ (first run, $\tau=0.509967$ ) and $\nu_{2}=1.66113 \times 10^{-2}$ (second run, $\tau=0.549834$ ). Figure 14 shows the contact line radius of the simulation over time. Both the FD and the LSQR model seem to recapture approximately Eq. (20), however, with a smaller exponent $<0.5$. This is acceptable, considering the low grid resolution and the static contact line model. The power law obtained, $r \sim t^{0.35}$, seems similar to the one reported in [56] for level set-based simulations.

\section{CONCLUSION}

Three different ways to compute the interface curvature from a VOF indicator function have been realized for comparison within a free surface LBM. A stationary Laplace bubble benchmark shows that methods based on geometric reconstruction (TR and LSQR, in the present study) can reach a significantly higher accuracy than continuum surface force 
approaches that are based on finite-difference approximations. In accordance with previous studies, reconstruction-based methods significantly reduce the magnitude of spurious currents. The LSQR approach shows a second-order rate of convergence with respect to grid spacing, which could not be achieved with the other two approaches.

For the generalized Laplace bubble test in a moving frame of reference, a previous study [32] reports convergence of errors, using a combination of higher-order advection scheme and curvature reconstruction in a Navier-Stokes discretization. This behavior could not be reproduced with the present FSLBM. Our results indicate that this lack of convergence is caused by the simplified advection of the indicator function that does not take into account any geometry information. In particular, the advection scheme fails to converge in a simple uniform advection test, indicating a lower order of accuracy than previously reported for simple reconstructionbased schemes (typically first or second order, with SLICor PLIC-based advection in Ref. [57]). Not surprisingly, the method thus fails to converge in the Laplace benchmark when conducted in a moving frame of reference including advection of the interface. This means that a conclusion drawn in Ref. [58] must be corrected: More accurate curvature estimation does not necessarily improve the FSLBM in surface tension-driven flows, since (asymptotically) the dominant error is caused by the advection scheme. Furthermore, it turned out that, in the moving case, the curvature estimation by FD is less sensitive to errors introduced by the advection scheme than the reconstruction-based approaches (LSQR and TR).

We have successfully extended the LSQR model to adhesive boundaries and compared it to the FD model in several numerical test cases. The order of convergence decreases to one in the presence of adhesive boundaries. However, for the stationary case, the errors of the new approach are still significantly smaller then those of obtained with the nonreconstructive FD approach. In dynamic scenarios, like contact line spreading on wetting surfaces, both models can recapture the basic inertial power-law dynamics. However, similarly to the bulk dynamic case, the error situation changes. Because the LSQR method is more sensitive to errors stemming from the FSLBM advection than the FD scheme, simulations do not profit from the higher-order scheme.

We conclude that a major improvement to the FSLBM would consist in a replacement of the advection scheme. VOF advection based on geometric reconstruction is significantly more complex and was, for this reason, excluded from the present study. In principle, any known advection scheme for VOF indicator functions [3,4] could be used to replace the simplified advection scheme of the FSLBM [59]. Considering that the simulation of surface tension according to the presented TR and LSQR schemes is based on a PLIC scheme to reconstruct the interface geometry, switching to a PLIC-based VOF advection scheme would be a possible solution. A major benefit in accuracy can be expected.

\section{ACKNOWLEDGMENTS}

Parts of the presented results were obtained during a stay at the Technical University Eindhoven. S.B. thanks the graduate school of the cluster of excellence "Engineering of Advanced Materials (EAM)" and the "3TU Research Centre Fluid and Solid Mechanics" (J.M. Burgerscentrum) for financial support and the institute of "Mesoscopic Transport Phenomena" (Technical University Eindhoven) for their hospitality. Further thanks go to the Bayerische Forschungsstiftung and KONWIHR project waLBerla-EXA for financial support.
[1] C. Körner, M. Thies, T. Hofmann, N. Thürey, and U. Rüde, J. Stat. Phys. 121, 179 (2005).

[2] C. W. Hirt and B. D. Nichols, J. Comput. Phys. 39, 201 (1981).

[3] R. Scardovelli and S. Zaleski, Annu. Rev. Fluid Mech. 31, 567 (1999).

[4] G. Tryggvason, R. Scardovelli, and S. Zaleski, Direct Numerical Simulations of Gas-Liquid Multiphase Flows (Cambridge University Press, Cambridge, 2011).

[5] R. Benzi, S. Succi, and M. Vergassola, Phys. Rep. 222, 145 (1992).

[6] S. Chen and G. D. Doolen, Annu. Rev. Fluid Mech. 30, 329 (1998).

[7] C. K. Aidun and J. R. Clausen, Annu. Rev. Fluid Mech. 42, 439 (2010).

[8] D. Wolf-Gladrow, Lattice-Gas Cellular Automata and Lattice Boltzmann Models: An Introduction (Springer, Berlin, 2005).

[9] S. Succi, The Lattice Boltzmann Equation for Fluid Dynamics and Beyond (Oxford Science Publications, Oxford, 2001).

[10] A. E. P. Veldman, J. Gerrits, R. Luppes, J. A. Helder, and J. P. B. Vreeburg, J. Comput. Phys. 224, 82 (2007).

[11] R. R. Nourgaliev, T. N. Dinh, T. G. Theofanous, and D. Joseph, Int. J. Multiphase Flow 29, 117 (2003).
[12] H. Liu, Q. Kang, C. R. Leonardi, S. Schmieschek, A. Narváez, B. D. Jones, J. R. Williams, A. J. Valocchi, and J. Harting, Comput. Geosci. 1 (2015).

[13] A. K. Gunstensen, D. H. Rothman, S. Zaleski, and G. Zanetti, Phys. Rev. A 43, 4320 (1991).

[14] X. Shan and H. Chen, Phys. Rev. E 47, 1815 (1993).

[15] M. R. Swift, W. R. Osborn, and J. M. Yeomans, Phy. Rev. Lett. 75, 830 (1995).

[16] U. Frisch, D. d'Humieres, B. Hasslacher, P. Lallemand, Y. Pomeau, and J.-P. Rivet, Complex Syst. 1, 649 (1987).

[17] M. Junk, A. Klar, and L.-S. Luo, J. Comput. Phys. 210, 676 (2005).

[18] D. J. Holdych, D. R. Noble, J. G. Georgiadis, and R. O. Buckius, J. Comput. Phys. 193, 595 (2004).

[19] X. Q. Xing, D. L. Butler, and C. Yang, Int. J. Numer. Methods Fluids 53, 333 (2007).

[20] D. Anderl, M. Bauer, C. Rauh, U. Rüde, and A. Delgado, Food Funct. 5, 755 (2014).

[21] E. Attar and C. Körner, Int. J. Heat Fluid Flow 32, 156 (2011).

[22] O. Svec, J. Skocek, H. Stang, M. R. Geiker, and N. Roussel, J. Non-Newtonian Fluid Mech. 179-180, 32 (2012).

[23] R. Ammer, M. Markl, U. Ljungblad, C. Körner, and U. Rüde, Comput. Math. Appl. 67, 318 (2014). 
[24] C. F. Janssen, S. T. Grilli, and M. Krafczyk, Comput. Math. Appl. 65, 211 (2013).

[25] C. Janssen, S. T. Grilli, and M. Krafczyk, in Proceedings of the Twentieth International Offshore and Polar Engineering Conference Beijing, China, June 20-25, 2010, edited by T. I. S. of Offshore and P. E. [The International Society of Offshore and Polar Engineers (ISOPE), Cupertino, CA, 2010], pp. 686-693.

[26] J. U. Brackbill, D. B. Kothe, and C. Zemach, J. Comput. Phys. 100, 335 (1992).

[27] B. Lafaurie, C. Nardone, R. Scardovelli, S. Zaleski, and G. Zanetti, J. Comput. Phys. 113, 134 (1994).

[28] D. Fuster, G. Agbaglah, C. Josserand, S. Popinet, and S. Zaleski, Fluid Dynam. Res. 41, 065001 (2009).

[29] M. M. Francois, S. J. Cummins, E. D. Dendy, D. B. Kothe, J. M. Sicilian, and M. W. Williams, J. Comput. Phys. 213, 141 (2006).

[30] D. Gerlach, G. Tomar, G. Biswas, and F. Durst, Int. J. Heat Mass Transf. 49, 740 (2006).

[31] T. Pohl, High Performance Simulation of Free Surface Flows Using the Lattice Boltzmann Method, Ph.D. thesis, University of Erlangen-Nuremberg, 2007.

[32] S. Popinet, J. Comput. Phys. 228, 5838 (2009).

[33] A. Q. Raeini, M. J. Blunt, and B. Bijeljic, J. Comput. Phys. 231, 5653 (2012).

[34] S. Afkhami and M. Bussmann, Int. J. Numer. Methods Fluids 57, 453 (2008).

[35] M. Sussman and M. Ohta, in Free Boundary Problems, International Series of Numerical Mathematics, Vol. 154, edited by I. N. Figueiredo, J. F. Rodrigues, and L. Santos (Birkhäuser Basel, Boston, Berlin, 2007), pp. 425-434.

[36] S. J. Cummins, M. M. Francois, and D. B. Kothe, Comput. Struct. 83, 425 (2005).

[37] H. Liu, S. Krishnan, S. Marella, and H. S. Udaykumar, J. Comput. Phys. 210, 32 (2005).

[38] G. Tryggvason, B. Bunner, A. Esmaeeli, D. Juric, N. Al-Rawahi, W. Tauber, J. Han, S. Nas, and Y.-J. Jan, J. Comput. Phys. 169, 708 (2001).

[39] Y. H. Qian, D. d'Humieres, and P. Lallemand, Europhys. Lett. 17, 479 (1992).

[40] I. Ginzburg, F. Verhaeghe, and D. d'Humieres, Commun. Comput. Phys. 3, 427 (2008).
[41] S. Bogner, R. Ammer, and U. Rüde, J. Comput. Phys. 297, 1 (2015).

[42] B. J. Parker and D. L. Youngs, Two and Three Dimensional Eulerian Simulation of Fluid Flow with Material Interfaces, (Tech. Rep., UK Atomic Weapons Establishment, 1992).

[43] M. W. Williams, D. B. Kothe, and E. G. Puckett, in Fluid Dynamics at Interfaces (Cambridge University Press, New York, 1998), pp. 294-305.

[44] W. Rider and D. Kothe, J. Comput. Phys. 141, 112 (1998).

[45] G. Taubin, in Proceedings of the Fifth International Conference on Computer Vision, 1995 (IEEE, Cambridge, MA, 1995), pp. 902-907.

[46] S. Donath, Wetting Models for a Parallel High-Performance Free Surface Lattice Boltzmann Method, Ph.D. thesis, University of Erlangen-Nuremberg, 2011.

[47] E. Anderson, Z. Bai, C. Bischof, S. Blackford, J. Demmel, J. Dongarra, J. Du Croz, A. Greenbaum, S. Hammarling, A. McKenney, and D. Sorensen, LAPACK Users' Guide (Society for Industrial and Applied Mathematics, Philadelphia, PA, 1999).

[48] Y. Renardy and M. Renardy, J. Comput. Phys. 183, 400 (2002).

[49] C. Feichtinger, S. Donath, H. Köstler, J. Götz, and U. Rüde, J. Comput. Sci. 2, 105 (2011).

[50] D. J. E. Harvie, M. R. Davidson, and M. Rudman, Appl. Math. Model. 30, 1056 (2006).

[51] D. Anderl, S. Bogner, C. Rauh, U. Rüde, and A. Delgado, Comput. Math. Appl. 67, 331 (2014).

[52] A. Caboussat, Arch. Comput. Methods Eng. 12, 165 (2005).

[53] A.-L. Biance, C. Clanet, and D. Quéré, Phys. Rev. E 69, 016301 (2004).

[54] Y. Sui, H. Ding, and P. Spelt, Annu. Rev. Fluid Mech. 46, 97 (2014).

[55] M. Renardy, Y. Renardy, and J. Li, J. Comput. Phys. 171, 243 (2001).

[56] H. Ding and P. D. M. Spelt, J. Fluid Mech. 576, 287 (2007).

[57] J. J. E. Pilliod and E. G. Puckett, J. Comput. Phys. 199, 465 (2004).

[58] S. Donath, K. Mecke, S. Rabha, V. Buwa, and U. Rüde, Comput. Fluids 45, 177 (2010).

[59] C. Janssen and M. Krafczyk, Comput. Math. Appl. 59, 2215 (2010). 